\title{
Design of Wireless Sensor Network Module in Power Consumption Information Collection System Based on IPv6
}

\author{
Yujun Liu \\ Beijing Inspection and Quarantine Testing Center of \\ Beijing Entry-Exit Inspection and Quarantine Bureau \\ 100026 China \\ Hongyan Yin \\ Beijing Inspection and Quarantine Testing Center of \\ Beijing Entry-Exit Inspection and Quarantine Bureau \\ 100026 China
}

\begin{abstract}
As an infrastructure service application system in smart grid, information collection system of electricity consumption plays a major role in ensuring the safe operation of power and achieving the orderly use of electricity. The paper presents a new scheme of wireless sensor networks based on Ipv6. In the paper, the designed information collection system includes the master station, channel, concentrators, collection, meter and so on. In the power consumption information collection system, the hardware and software research of concentrators, routers and wireless module are very important. In this paper, the hardware and software are designed based on IPv6 wireless sensor module, including structural design, interface design, data structure design and operation design of built-in wireless communication concentrator module.
\end{abstract}

Keywords- Wireless Sensor Network; IPv6; Information collection system

\section{INTRODUCTION}

In the smart grids, power consumption information collection system is a very important part to ensure the safe operation of power and achieve the orderly use of electricity. In such a system, communication networks are very important. There are many communication systems can be used, such as wire-based or wireless-based communication [1]. In the wire-based communication systems, powerline communication is with the unique networks and is used as the backbone for the information collection systems [2]. For the wireless-based communication systems, the wire less senor networks seem to be the optimal choice, because it has many characteristics, such as multi node, complex application scenarios. The short distance wireless communication technology can be applied to the local network, which is composed of a wireless ad hoc network through multiple collection terminals, and the data will be collected by multi hop manner sending to the concentrator. Wireless Sensor Network, WSN, has a very unique advantage, such as the nodes of large-scale deployment, flexible networking, low cost [3]. WSN is as an effective supplement to other networking mode has been widely used in smart grid for power consumption information collection system. WSN can realize transparent transmission of IoT (Internet of

\author{
Tong $\mathrm{Wu}$ \\ China National Institute of Metrology
}

things, IoT), seamless interconnection, plug and a full IP with end-to-end solutions.

In the power consumption information collection system, the hardware and software research of concentrators, routers and wireless module is very important. In this paper, the hardware and software are designed based on the IPv6 wireless sensor module, including structural design, interface design, data structure design and operation design of built-in wireless communication concentrator module. The focus of this paper are selecting the appropriate chip for the radio frequency of $470-510 \mathrm{MHz}$, adopting $802.15 .4 \mathrm{~g}$ in $\mathrm{PHY}$ and MAC, using 6LoWPAN protocol in upper.

\section{HARDWARE DESIGN OF WIRELESS MODULE}

The hardware configuration of wireless module is relatively compact and the wireless RF communication module in the master module is connected via bus communication interface with SPI (Serial Peripheral Interface). Thus the register realizes the functions of reading and writing [4] as well as the configuration of the module parameters. Further, wireless communication module can realize sending and receiving wire less data. The master module obtain the data which is transmitted by $\mathrm{RF}$ modules, then upload to the desired uplink interface through UART (Universal Asynchronous Receiver/Transmitter). The overall system design is shown in Fig. 1.

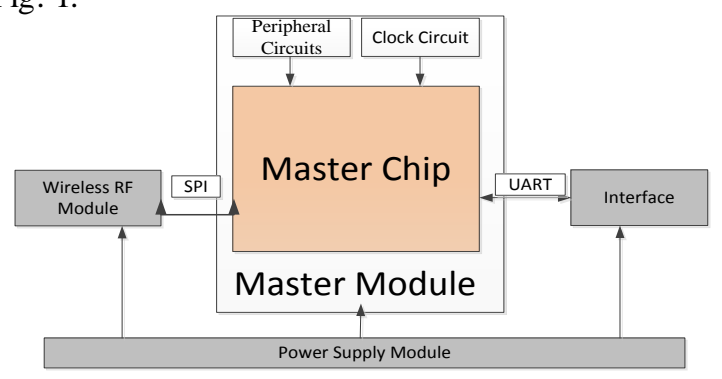

Figure 1. Overall design of wireless module

Because the chip of RF module is required to support IEEE802.15.4g communication protocol, so the selection of chips are SI4463 manufactured by Silicon labs [3]. The 
hardware structure and interface of wireless module are shown in Fig. 2.

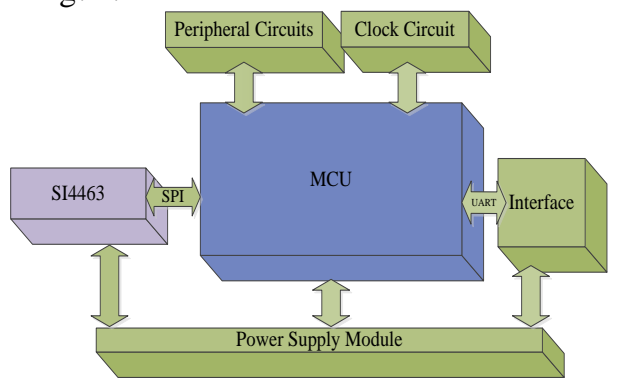

Figure 2. The hardware structure and interface of wireless module

We can breifly described every part in the structure.

(1) RF module (mainly based on the SI4463 RF chip) is mainly used to send and receive wireless data packets, including RF module design of the peripheral circuit;

(2) The peripheral circuit and clock circuit of MCU are to complete control of RF modules;

(3) Power supply modules provide modules with power, you need to evaluate power source for each module, in order to guarantee the system stable run with typical power value;

(4) UART mainly complete the data transfer between host computer, mainly sends the data which is acquired by the wireless module after processing by MCU to the host computer analysis software for the analys is of the wireless data by the host computer protocol software.

\section{THE HARDWARE DESIGN OF ROUTER}

Hardware configuration of the router include: control module, power supply module, RF module. The main function of the router is used to receive data which send by a wireless node, after gathering the data uploaded to the gateway. The overall systemsolution is shown in Fig. 3.

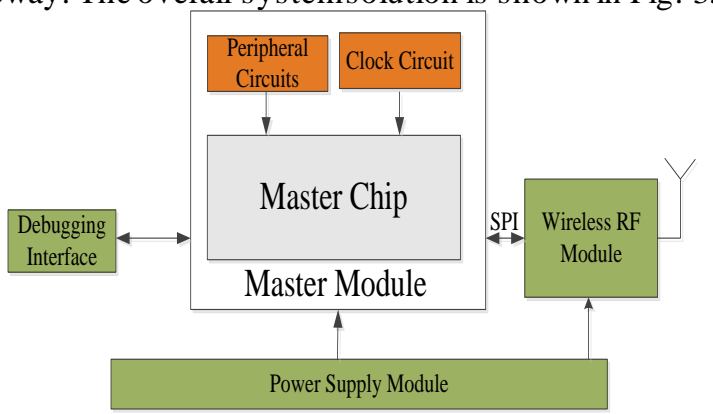

Figure 3. Overall design of router module

\section{THE HARDWARE DESIGN OF CONCENTRATOR}

Wireless concentrator adopts modular design, according to their functions, it is divided into: control circuit, wire less modules, Ethernet module, power module, serial module, LED. Data concentrator collects data on the lower collector via wireless transceiver module, then transfer to the control module for processing. In the end, the processed data will be send through an Ethernet interface to the host computer. Also, the concentrator can also respond to the host computer's instructions to complete the communication task. The hardware structure of concentrator is shown in Fig. 4.

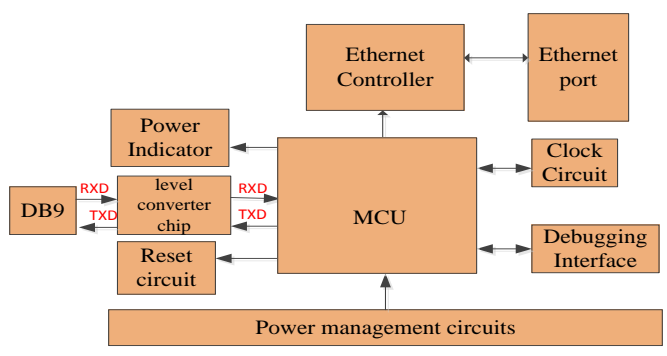

Figure 4. The hardware structure of concentrator

Concentrator module with automatic routing management capabilities, support for remote upgrades, built-in TCP / IP protocol. It is able to support network online upgrade, data acquisition and collection. When configure the central nodes and child nodes, we can utilize 6LoWPAN dynamic address allocation mechanism to assign each node a unique 16-bit short address in the PAN.

\section{SOFTWARE DESIGN OF METER CONCENTRATOR MODULE}

Concentrator modules of meter concentrator on business software platform, which provides basic data collection capabilities for supporting the upper services, supporting downlink communication driver, supporting data collection, and provide the corresponding interface for calls. Local acquisition and monitoring applications software can be further developed on this basis, coupled with application-specific graphical user interface (GUI), in order to achieve a complete meter system.

\section{A. Structural Design}

Hierarchy of the entire module is shown in Fig. 5.

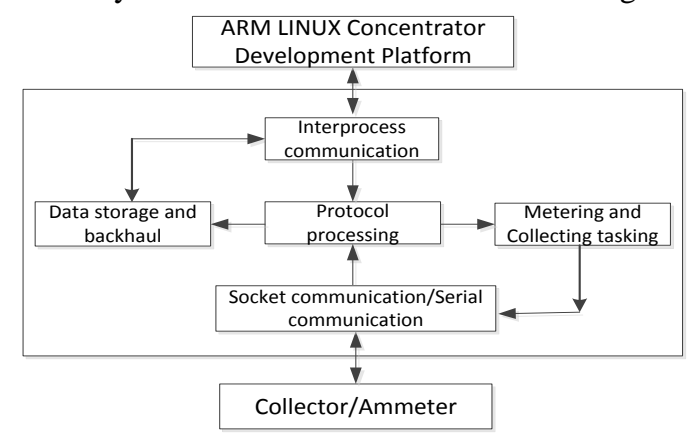

Figure 5. Hierarchy of the entire module

Module provides multi-task scheduling, channel acquisition management, data management and interprocess communication services. From the point of business functions, the module is divided into multiple sub-modules as follows: protocol processing module, interprocess communication module, Socket communication module, data dictionary management module, collecting task module, channeldetection module.

Software module division is according to real-time response and capabilities of cohesion [6], the performance of real-time response requires some modules are broken down into smaller sub-modules again, because the time request of the sub-module will be different, so they can be called in different tasks. Capabilities of cohesion require the module with high cohesion. The coupling between modules is small, so that the structure can ensure a clear 
interface between modules, thereby reducing the overall debugging difficult.

The main function modules realize data transmission and interaction through the list, each module calls a unified data access interface to read and write. Such data coupling between modules greatly reduces, and the errors to access the data have also reduced.

\section{B. Interface Design}

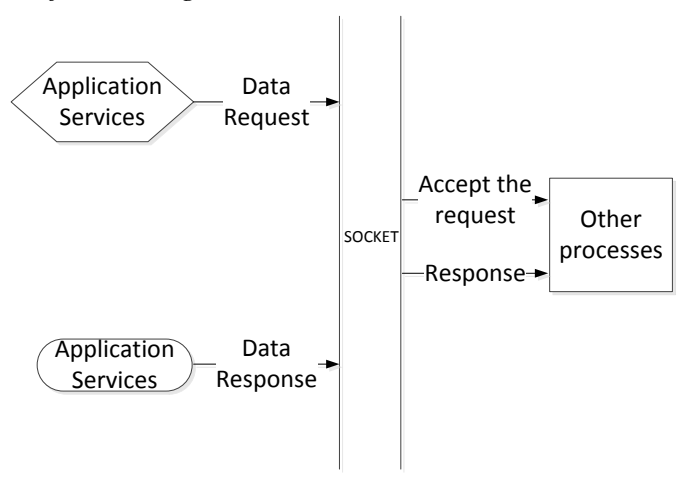

Figure 6. Interprocess communication interface

The interfaces which are used to communicate software with Master Scheduling for linking channel, closing channel, transceiver and handling exception data. Communication protocol uses a custom extension agreement. The design adopts unified data interface and module has its own unique channel ID, which is shown in Fig. 6.

It is responsible for managing and associating meter archives and collector archives. Data structures are regarded the collector as a management unit. Collector belongs to meter. Both of them are identified a unique ID by collector. It manages meter tasks and related parameters Furthermore, get the data from the system to make the appropriate filter according to collecting mechanisms. It manages the real-time data collected by meter and timing data meter. It regards the meter as a management unit. Power meter includes all the data items collected by itself. It manages collected information and collector connection information. The returned data is made into a unified cache handling, and then follow the statute of the data structure to further processing. It manages the data collected by collector, mainly for concurrent collecting. In the overall operation of the software, it begins to run from the main function, carried by the $\mathrm{C}$ compiler to start the program to call the main function. Realize the process in the main function to initialize the process, create the semaphore, monitor task creation, start mu lti-task and other operations. The overall operation of the software process is shown in Fig. 7.

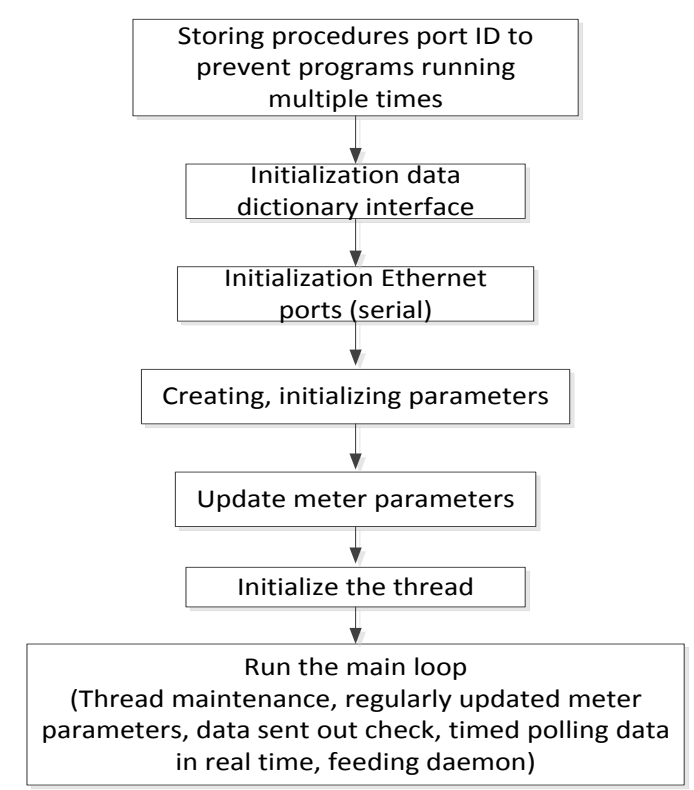

Figure 7. The overall operation of the software process

\section{CONCLUSIONS}

The paper introduces the hardware design of IPv6based wireless sensor module, including routers and concentrators module design, and the software of meter modules, including structural design, interface design, data structure design and operation design.

\section{ACKNOWLEDGMENT}

This work is supported by the national quality ins pection service project under Grant 201410050.

The "SmartSignalProcessing" software is used to process the signals from the smart meters. Such software is developed by Prof. Ming-Yue ZHAI with North China Electric Power University.

\section{REFERENCES}

[1] ZHAI Ming-Yue, Transmission Characteristics of Low-Voltage Distribution Networks in China Under the Smart Grids Environment. IEEE Transaction on Power Delivery. Vol. 26, No. 1, JANUARY 2011,pp.173-180.

[2] ZHAI Ming-Yue. Signal Recovery in Power Line Communications Systems Based on the Fractals. IEEE Transaction on Power Delivery. Vol. 26, No. 3, July 2011, pp.1864-1872.

[3] S.Amin, B.Wollenberg. Toward a smart grid[J]. IEEE Power and Energy Magazine, 2005,31(3):34-41

[4] Pao-Hsiang His, Shi-Lin Chen. Distribution Automation Communication Infrastructure[J]. IEEE Transaction on Power Delivery, 2008,V13(3):728-734

[5] GUNGOR V C, LAMBERT F C. A survey on communication networks for electric system automation[J].Computer Networks.2006,50(3):877-897

[6] Kermajani Hamidreza, Gómez Carles. Contributions to the performance evaluation and improvement of the IPv6 routing protocol for low-power and lossy networks[D]. Universitat Politècnica De Catalunya, 2014. 